\title{
SMALL COMETS: IMPLICATIONS FOR INTERPLANETARY LYMAN $\alpha$
}

\author{
T. M. Donahue
}

\section{Department of Atmospheric and Oceanic Science, Space Physics Research Laboratory, The University of Michigan}

Abstract. If small comets are as numerous in the inner solar system as Frank et al. [1986a] have recently proposed, large and easily detectable amounts of hydrogen would be generated in interplanetary space. Inhibition of water vapor production by a factor of $3 \times 10^{-9}$ by very thick dust mantles would be necessary to prevent excitation of a detectable interplanetary Lyman $\alpha$ glow. Other optical problems associated with the cometesimals are also discussed.

Frank et al. [1986a], henceforth FSC, have proposed that small comets 12 meters in diameter, whose density is $0.1 \mathrm{gm} \mathrm{cm}^{-3}$, impact the earth at a rate of about $20 \mathrm{per}$ minute. This rate requires that the average distance between these cometesimals at $1 \mathrm{AU}$, before gravitational focussing by the earth, be about $5000 \mathrm{~km}$ and their spatial density be about $3 \times 10^{-26}$ comets $\mathrm{cm}^{-3}$ [Frank, et al., 1986b]. The "standard" rate for production of water molecules from comets at $1 \mathrm{AU}$ is between $10^{17}$ and $10^{18} \mathrm{~cm}^{-2} \mathrm{~s}^{-1}$ or $3 \times 10^{24}$ molecules every second from each small comet. Dissociation of the water would result in the production of hydrogen atoms at a rate of $0.14 \mathrm{~cm}^{-3} \mathrm{~s}^{-1}$ from this family of small comets at 1 $\mathrm{AU}$, if the water production rate is $5 \times 10^{17} \mathrm{~cm}^{-2} \mathrm{~s}^{-1}$. If the lifetime of these atoms before charge exchange with the solar wind ions is $2 \times 10^{6}$ seconds, their density near $1 \mathrm{AU}$ would be about $3 \times 10^{5}$ per $\mathrm{cm}^{3}$. FSC, recognizing the problem of loading the solar wind with so many pick-up ions, proposed that the rate of gas production from the small comets must be kept low by sufficiently thick dust mantles on their exteriors. Reducing the production rate by orders of magnitude, to a level between $3 \times 10^{10}$ and $3 \times 10^{11} \mathrm{~cm}^{-2} \mathrm{~s}^{-1}$, would avoid the difficulty with excess generation of pick-up ions [Frank, et al., 1986b]. It would not be sufficient to escape another difficulty - the excitation of Lyman $\alpha$ radiation by the cometary hydrogen. If gas generation is assumed to vary with heliocentric distances as $r^{-2.6}$ [Meier and Keller, 1985], a column perpendicular to the solar direction at $1 \mathrm{AU}$ would contain $4.5 \times 10^{18}$ atoms cm $\mathrm{cm}^{-2}$ and have an optical depth of $7 \times 10^{5}$ for Lyman $\alpha$, a fact already pointed out by Chubb [1986]. Interplanetary hydrogen is not optically thick in the inner solar system [Purcell and Tousey, 1960; Meier and Prinz, 1970].

The interplanetary Lyman $\alpha$ glow at $1 \mathrm{AU}$ is about $400 \mathrm{R}$ at solar minimum and $1.2 \mathrm{kR}$ at solar maximum. If the specific rate at which solar Lyman $\alpha$ radiation is scattered by hydrogen atoms at $1 \mathrm{AU}$ is taken as $1 \times 10^{-3} \mathrm{~s}^{-1}$ at solar minimum and $3 \times 10^{-3} \mathrm{~s}^{-1}$ at solar maximum [Hinteregger, 1981; Mount, et al., 1980], and the water production rate from small comets is $4 \times 10^{10}$ molecules $\mathrm{cm}^{-2} \mathrm{~s}^{-1}\left(10^{-7}\right.$ times normal), the entire interplanetary glow would be ascribable to small comets. This interplanetary glow would be confined to the inner solar system. Observations performed

Copyright 1987 by the American Geophysical Union.

Paper number 6L6485.

$0094-8276 / 87 / 006 \mathrm{~L}-6485 \$ 03.00$ by ultraviolet instruments on Pioneer 10 and Voyagers 1 and 2 have demonstrated that this is not the case. The gas production rate set by FSC to prevent mass loading of the solar wind would result in Lyman $\alpha$ emission rates between 250 and 750R (solar minimum and solar maximum) for the lowest part of its range $\left(3 \times 10^{10} \mathrm{~cm}^{-2} \mathrm{~s}^{-1}\right)$. A contribution to the interplanetary Lyman $\alpha$ radiation at $1 \mathrm{AU}$ of as much as $25 \mathrm{R}$ by the hydrogen associated with small comets would have been detected as a signal rapidly decreasing with heliocentric distance by the ultraviolet spectrometers on Voyager 1 and 2. The FSC comets could emit water molecules at a rate no larger than $1.3 \times 10^{9} \mathrm{~cm}^{-2} \mathrm{~s}^{-1}\left(3 \times 10^{-9}\right.$ times the normal value for comets) if their contribution to interplanetary Lyman $\alpha$ is not to exceed $25 \mathrm{R}$ at $1 \mathrm{AU}$, when the specific excitation rate for Lyman $\alpha$ is $2 \times 10^{-3} \mathrm{~s}^{-1}$. This limit is 0.04 times as large as the smaller of the limits set by FSC to avoid mass loading of the solar wind.

FSC have invoked a model for cometary mantles developed by Fanale and Salvail [1984] to demonstrate that gas production rates can be kept as low as $3 \times 10^{11} \mathrm{~cm}^{-2} \mathrm{~s}^{-1}$ at $1 \mathrm{AU}$, if the cometesimals have dust mantles whose thickness is $1 \mathrm{~cm}$ or less. As Morris [1986] has already pointed out, there is a serious problem with such comets retaining a mantle. Apart from this issue, the Fanale and Salvail model in fact does predict that the gas production rate will be between $3 \times 10^{10}$ and $3 \times 10^{11}$ molecules $\mathrm{cm}^{-2} \mathrm{~s}^{-1}$ if the mantle thickness is between 15 and $1.5 \mathrm{~cm}$. To drop the production rate another factor of 25 to meet the Lyman $\alpha$ constraint, the mantle would need to be 3.75 meters thick - and the cometesimal mostly mantle - according to the model of Fanale and Salvail.

It is important to notice that the model of Fanale and Salvail leads to production rates for a given mantle thickness that are orders of magnitude lower than those obtained by others [Gombosi, et al., 1986]. This seems to be due to the assumption of an extraordinarily small value for the radius of the mantle capillaries through which the escaping gas must flow (half the average dust grain radius or less than $1 \mu \mathrm{m}$ ), as Gombosi, et al. [1986] point out. More typical of other models is that of Horanyi, et al [1984], where the characteristic capillary dimension is that of the intergrain distance. When mantles are thick, the gas flows predicted by other models tend to be as much as four orders of magnitude greater than those of Fanale and Salvail for a given mantle thickness. For example, a calculation by Gombosi (private communication, 1987), using the Horányi, et al. [1984] model, requires that the mantle be $750 \mathrm{~m}$ thick to hold the gas production rate to $3 \times 10^{11} \mathrm{~cm}^{-2} \mathrm{~s}^{-1}$ and $172 \mathrm{~km}$ thick to keep it at $1.3 \times 10^{9} \mathrm{~cm}^{-2} \mathrm{~s}^{-1}$, as the interplanetary Lyman $\alpha$ limit requires. Whatever mantle theory is valid, the influx of dust into the earth's atmosphere accompanying the FSC cometesimals would appear to be forbiddingly large, as Rubicam [1986] and Morris [1986] have maintained. The fluxes would be very much larger than those discussed by FSC [1986b, 1986d] in their reply to the comments of Ru- 
bicam and Morris. The carbon influx, for example, would be more than 350 times as large, far exceeding the rate of burial of fresh carbon in sediments. Except in the case of the Fanale and Salvail model, a cometoid mantle thick enough to supress excess Lyman $\alpha$ excitation in the inner solar system does not appear to be physically attainable. If it were, the dust influx into the earth's atmosphere would be intolerably large. Even relaxing the Lyman $\alpha$ condition by a factor of 4 ( $100 \mathrm{R}$ contribution to the interplanetary Lyman $\alpha$ glow at 1 AU) would not materially affect these conclusions.

According to FSC [Frank, et al., 1986c], the water vapor released from cometoids striking the moon would cause the moon to be surrounded by a hydrogen corona similar to that around an ordinary comet. This would be the consequence of the remarkable, almost perfectly elastic, collisions of small comets with the moon for which they argue. The collisions would result in rapid outstreaming of the water vapor associated with the cometary ice. They predict that the emission rate of solar Lyman $\alpha$ radiation scattered by this lunar hydrogen coma would be about $80 \mathrm{R}$, when observed from afar, in a direction tangent to the lunar surface, at the subsolar point. Fastie, et al., [1973] repeatedly measured the emission rate of Lyman $\alpha$ from the Apollo 17 service module as it was orbiting at an altitude 45 to $70 \mathrm{~km}$ above the lunar subsolar point, with the direction of the field of view of their spectrophotometer perpendicular to the direction of the sun. The emission rate measured in this way, just as the service module was beginning its return to earth, was $196 \mathrm{R}$. The counting error in this measurement was negligible ( $1 \mathrm{R})$. The field of view ( $68^{\circ}$ R.A. and $\left.26^{\circ} \mathrm{dec}\right)$ was in the direction of minimum interplanetary emission. Because the contribution of starlight to this measurement is uncertain by $5 R$, the brightness of the diffuse sky background was $196_{-5}^{+1} R$. According to FSC [1986], 40 R of this emission would be ascribable to the lunar Lyman $\alpha$ glow, leaving only about $155_{-5}^{+1} \mathrm{R}$ attributable to the interstellar wind. This is much lower than values obtained from other spacecraft at times of similar solar activity - about $250 \mathrm{R}$. The Apollo 17 spectrometer recorded geocoronal Lyman $\alpha$ emission rates comparable to those measured by other instruments under similar conditions, so it does not seem likely that a serious calibration problem would explain this discrepancy.

The interplanetary Lyman $\alpha$ glow was monitored almost continuously from the service module during transearth coast. Since the lunar glow has a scale length of about $10^{6} \mathrm{~km}$, according to FSC, its brightness should have increased to about $50 \mathrm{R}$ when the service module was entering the geocorona at a distance of $10^{5} \mathrm{~km}$ from Earth. Unfortunately, the uncertainties involved in accounting for stellar Lyman $\alpha$ and solar Lyman $\alpha$ variability exceed the $10 \mathrm{R}$ variation that would occur because of the lunar hydrogen halo. Hence these observations probably cannot be used to determine whether the water from the cometesimals issues from the moon at the rate claimed by FSC.

Finally, it is interesting that a comet $12 \mathrm{~m}$ in diameter $1700 \mathrm{~km}$ above the earth would be observable to the naked eye in twilight as a moving object brighter than 6 visual magnitude, unless its surface reflectivity is less than 15 percent. The reflectivity would need to be less than 0.15 percent to preclude detection with a $75 \mathrm{~mm}$ diameter monocular telescope. This is 0.04 of the Fresnel reflection coefficient of a smooth sphere at $45^{\circ}$. A physical light trap to achieve this condition could be built, but its dimensions would be very much larger than those of dust-grains (W. G. Fastie, personal communication, 1987).

Thus, there are two reasons that these remarkable comets would need a dark protective covering: one is to suppress water vapor production by a factor of $3 \times 10^{-9}$ in order to render them unobservable at $1216 \AA$, and the other is to make them invisible to comet hunters. In neither case does it appear to be physically possible to achieve the conditions required.

Acknowledgements. I wish to thank William G. Fastie and Tamas Gombosi for their interest and help. L. A. Frank made very useful comments regarding the interpretation of the Apollo 17 observations. This material is based on work supported by the National Science Foundation under Grant No. ATM-852 0653.

\section{References}

Chubb, T. A., Comment on the paper "On the influx of small comets into the earth's upper atmosphere, I. Observations", Geophys. Res. Lett., 13, 1075-1077, 1986.

Fanale, F. P. and J. R. Salvail, An idealized short-period comet model: Surface insolation, $\mathrm{H}_{2} \mathrm{O}$ flux, dust flux and mantle evolution, Icarus, 60, 476-511, 1984.

Fastie, W. G., P. D. Feldman, R. C. Henry, H. W. Moos, C. A. Barth, G. E. Thomas, and T. M. Donahue, A search for far-ultraviolet emissions from the lunar atmosphere, Science, 182, 710-714, 1973.

Frank, L. A., J. B. Sigwarth, and J. D. Craven, On the influx of small comets into the Earth's upper atmosphere, II. Interpretation, Geophys. Res. Lett., 13, 307-310, 1986a.

Frank, L. A., J. B. Sigwarth, and J. D. Craven, Reply, Geophys. Res. Lett., 13, 703-704, 1986 b.

Frank, L. A., J. B. Sigwarth, and J. D. Craven, Reply to Davis and Nakamura, et al., Geophys. Res. Lett., 13, 1186-1189, 1986c.

Frank, L. A., J. B. Sigwarth, and J. D. Craven, Reply, Geophys. Res. Lett., 13, 1484-1486, 1986d.

Gombosi, T. I., A. F. Nagy, and T. E. Cravens, Dust and neutral gas modeling of the inner atmosphers of comets, Rev. Geophys., 24, 667-700, 1986.

Hinteregger, H. E., Representation of solar EUV fluxes for aeronomical applications, Adv. Space Res., 1, 39-52, 1981.

Horányi, M., T. I. Gombosi, T. E. Cravens, A. Korosmezey, K. Kecskemety, A. F. Nagy, and K. Szego, The friable sponge model of cometary nuclei, Astrophys. J., 278, 449 455, 1984.

Meier, R. R., and H. U. Keller, Predictions of the hydrogen lyman alpha coma of Comet Halley, Icarus, 62, 521-536, 1985.

Meier, R. R., and D. K. Prinz, Absorption of the solar lyman line by geocoronal atomic hydrogen, J. Geophys. Res., 75, 6969-6979, 1970.

Morris, D. E., Comment on "On the influx of small comets into the Earth's upper atmosphere, II. Interpretation", Geophys. Res. Lett., 13, 1482-1483, 1986.

Mount, G. H., G. J. Rottman, and J. G. Timothy, The solar spectral irradiance $1200-2500 \AA$ at solar minimum, $J$. Geophys. Res., 85, 4271-4274, 1980.

Purcell, J. D., and R. Tousey, The profile of Solar Lyman 
Alpha, in Space Research, edited by H. Kallmann Bijl, pp. 590-593, North-Holland Publishing Co., Amsterdam, 1960.

Rubicam, D., Comment on "On the influx of small comets into the Earth's upper atmosphere, II. Interpretation", Geophys. Res. Lett., 13, 701-702, 1986.
T. M. Donahue, Space Physics Research Laboratory, Atmospheric and Oceanic Science Department, The University of Michigan, Ann Arbor, MI 48109-2143.

(Received December 22, 1986; accepted January 12, 1987.) 